Applied Physiology, Nutrition, and Metabolism Physiologie appliquée, nutrition et métabolisme

\title{
The treatment effects of flaxseed-derived secoisolariciresinol diglycoside and its metabolite enterolactone on benign prostatic hyperplasia involve the $\mathbf{G}$ protein-coupled estrogen receptor 1
}

\begin{tabular}{|r|l|}
\hline Journal: & Applied Physiology, Nutrition, and Metabolism \\
\hline Manuscript ID & apnm-2016-0332.R2 \\
\hline Manuscript Type: & Article \\
\hline Date Submitted by the Author: & $12-$ Sep-2016 \\
\hline Complete List of Authors: & $\begin{array}{l}\text { Ren, Guan-Yu; Soochow University } \\
\text { Chen, Chun-Yang; Soochow University } \\
\text { Chen, Wei-Guo; Soochow University } \\
\text { Huang, Ya; Soochow University } \\
\text { Qin, Li-Qiang; Soochow University } \\
\text { Chen, Li-Hua; School of Public Health, Soochow University; , Department } \\
\text { of Nutrition and Food Hygiene }\end{array}$ \\
\hline Keyword: & $\begin{array}{l}\text { Flaxseed, Lignan, Benign prostatic hyperplasia, G Protein-Coupled Estrogen } \\
\text { Receptor 1 }\end{array}$ \\
\hline
\end{tabular}


The treatment effects of flaxseed-derived secoisolariciresinol diglycoside and its metabolite enterolactone on benign prostatic hyperplasia involve the $G$ protein-coupled estrogen receptor 1

Guan-Yu Ren ${ }^{1,2, \#}$, Chun-Yang Chen ${ }^{1,2, \#}$, Wei-Guo Chen ${ }^{2}$, Ya Huang ${ }^{3}$, Li-Qiang Qin ${ }^{1}$, Li-Hua Chen ${ }^{1, *}$

${ }^{1}$ Jiangsu Key Laboratory of Preventive and Translational Medicine for Geriatric Diseases, Department of Nutrition and Food Hygiene, School of Public Health, Soochow University;

199 Renai Road, Dushu Lake Higher Education Town, Suzhou 215123, China;

Email for Guan-Yu Ren: 15850177665@163.com;

Email for Chun-Yang Chen: chen20154232027@126.com;

Email for Li-Qiang Qin: qinliqiang@suda.edu.cn;

${ }^{2}$ Department of Urology ,The First Affiliated Hospital of Soochow University, Soochow University; 188 Shizi street, Suzhou 215006, China; Email for Wei-Guo Chen:15312172967@163.com;

${ }^{3}$ Institute of Neuroscience, Soochow University;

199 Renai Road, Dushu Lake Higher Education Town, Suzhou 215123, China;

Email for Ya Huang: yhuang@suda.edu.cn ;

- Word counts: Abstract: 243; The text (excluding references and figures):3997 
\# Indicates these authors contribute equally to the work

* Indicates corresponding author; E-mail: chenlihua@suda.edu.cn; Tel: +86

512-65880075; Fax: +86 512-65883323 


\begin{abstract}
Secoisolariciresinol diglucoside (SDG), a lignan extracted from flaxseed, has been shown to suppress benign prostatic hyperplasia (BPH). However, little is known about the mechanistic basis for its anti-BPH activity. The present study showed that enterolactone (ENL), the mammalian metabolite of SDG, shared the similar binding site of G1 on a new type membranous estrogen receptor-G Protein-Coupled Estrogen Receptor 1 (GPER) by docking simulations method. ENL and G1 (the specific agonist of GPER) inhibited the proliferation of human prostate stromal cell line WPMY-1 showed by MTT assay and arrested cell cycle at the G0/G1 phase displayed by propidium iodide staining following flow cytometer examination. Silencing GPER by siRNA attenuated the inhibitory effect of ENL on WPMY-1 cells. The therapeutic potential of SDG in the treatment of $\mathrm{BPH}$ was confirmed in a testosterone propionate-induced BPH rat model. SDG significantly reduced the enlargement of the rat prostate and the number of papillary projections of prostatic alveolus and thickness of the pseudostratified epithelial and stromal cells when comparing with the model group. Mechanistic studies showed that SDG and ENL increased the expression of GPER both in vitro and in vivo. Furthermore, ENL-induced cell cycle arrest may be mediated by the activation of GPER/ERK pathway and subsequent up-regulation of p53 and p21, down-regulation of cyclin D1. This work, in tandem with previous studies, will enhance our knowledge regarding the mechanism(s) of dietary phytochemicals on BPH prevention and ultimately expand the scope of adopting alternative approaches in $\mathrm{BPH}$ treatment.
\end{abstract}


Keywords: Flaxseed; Lignan; Benign prostatic hyperplasia; G Protein-Coupled

Estrogen Receptor 1 


\section{Introduction}

Benign prostatic hyperplasia (BPH) is a progressive age-related pathologic proliferation of prostatic glandular and stromal tissues (Isaacs 1994). BPH is clinically characterized by urinary frequency, urgency, nocturia, decreased and intermittent force of stream, and sensation of incomplete bladder emptying. There is no completely effective treatment for BPH. Medical therapies include $\alpha$-adrenergic blockers, $5 \alpha$-reductase inhibitors (5-ARIs) and their combinations. Although evidence from clinical practice has proven the efficacy of these agents in providing symptomatic benefit, a plethora of side effects is associated with the use of these drugs. Surgery, usually transurethral prostate resection, is currently the most effective intervention for $\mathrm{BPH}$, but it is also associated with a multitude of postoperative systemic complications commonly referred to as the TURP syndrome (Ventura et al. 2011). Thus, exploration of safer and more economical treatments are clearly needed.

Recently, flaxseed and its derivative secoisolariciresinol diglucoside (SDG) are increasingly used in the human diet because of its potential benefits, including cardiovascular protection(Pan et al. 2009; Ren et al. 2016; Wu et al. 2010) and prostate health enhancement(Azrad et al. 2013; Demark-Wahnefried et al. 2008a;

Demark-Wahnefried et al. 2008b; Demark-Wahnefried et al. 2001;

Demark-Wahnefried et al. 2004). A recent double-blind, placebo-controlled study by Simons et al.(Simons et al. 2015) found that supplementation with flaxseed hull 
extract (equivalent to100 mg, $200 \mathrm{mg}$ SDG) was safe and well-tolerated and improved the quality of life of individuals with BPH. Moreover, a high quality randomized controlled trial (RCT) conducted by Zhang et al.(Zhang et al. 2008) showed that comparing with placebo, SDG supplementation (300 mg, $600 \mathrm{mg}$ ) significantly decreased the International Prostate Symptom Score (IPSS), improved the Quality of Life score (QOL score), alleviated lower urinary tract symptoms (LUTS). More importantly, the therapeutic efficacy appeared comparable to that of commonly used intervention agents of $\alpha$-adrenergic blockers and 5-ARIs(Zhang et al. 2008). Although evidence from clinical trials has proved the beneficial effects of SDG on BPH alleviation, the underlying mechanisms are unclear.

SDG, a kind of lignans, which are hydroxylated bicyclobenzylbutane diol derivatives. After consumption, SDG is converted by the intestinal microbiota to mammalian lignan of enterolactone (ENL), the major form in the biological fluids of humans and animals(Borriello et al. 1985). ENL is a polyphenol compound and its chemical structure is similar with estradiol. Estrogen can modulate cellular physiology by both genomic and non-genomic pathways. Many of its rapid, non-genomic effects have been suggested to be mediated via the activation of a new type membranous estrogen sensitive G-protein coupled receptor (G-protein-coupled estrogen receptor 1, GPER; or formerly known as GPR30)(Gencel et al. 2012). Activation of GPER leads to c-SRC-dependent trans-activation of the epidermal growth factor receptor (EGFR) and consequent stimulation of ERK 1/2 (Filardo et al. 2000) and PI3K/AKT 
pathways (Meyer et al. 2014). Previously, we demonstrated that ENL suppressed IGF-1 induced proliferation of prostate cancer cells by inhibiting its downstream AKT phosphorylation(Chen et al. 2009). As membrane receptors initiate cascades of phosphorylation events(Smith et al. 2016), we are wondering whether ENL could interact with GPER.

In this study, we showed the inhibitory role of ENL in the growth of BPH both in vitro and in vivo by investigating the effects of ENL on GPER and its downstream ERK1/2 kinase activation cascade that is causally linked to up-regulation of p21, and cell-cycle arrest at the G1 phase.

\section{Materials and methods}

\section{Chemicals and reagents}

Testosterone propionate (TP) was purchased from Shanghai general pharmaceutical co., LTD (Shanghai, China). SDG was from Biopurify Phytochemicals (Chengdu, China). G-1 is from Cayman Chemical (Ann Arbor, MI, USA). We also purchased antibodies from Cell Signaling Technology (phospho-ERK, Tublin, Actin, P21 and Cyclin D1; Shanghai Division, China) and Santa Cruz Biotechnology (GPR30, GPR30 siRNA; Shanghai Division, China). Enterolactone, Finasteride, 3-(4,5dimethylthiazol-2-yl)-2,5-diphenyltetrazolium bromide (MTT) and other chemicals that were not specifically indicated were purchased from Sigma (Shanghai Division, China).

\section{Molecular modeling and docking simulations}


All the molecular structures of G-1 and ENL were screened "in silico". To analyse the potential interaction between GPER and ENL, we build a model of GPER by homology and operate the program on Discovery Studio 4.0 Modeler (Biovia, Inc., San Diego, CA). Bovine rhodopsin (PDB code 1U19) sharing 40\% amino acid sequence with GPER, was choose as the template to build GPER model (Lappano et al. 2012). The homology structure of the GPER was analyzed and verified with Profile-3D and Ramachandran plot following the method as described previously (Yu et al. 2013). The resulting model was used as target for molecular docking simulations using CDOCKER module embedded in the Discover Studio 4.0 (Wu et al. 2003).

\section{Cell culture}

The human prostatic stromal cell line, WPMY-1 was obtained from the American Type Culture Collection (ATCC, Manassas, VA, USA). WPMY-1 cells were routinely cultured in DMEM supplemented with 10\% fetal bovine serum, $10 \mathrm{mmol} / \mathrm{L}$ HEPES, $1 \%$ penicillin, and streptomycin (GIBCO BRL). The cells were maintained at $37{ }^{\circ} \mathrm{C}$ in a 5\% CO2 humidified incubator. ENL was dissolved in dimethyl sulfoxide (DMSO) at a concentration of $100 \mathrm{mmol} / \mathrm{L}$ and stored at $-20{ }^{\circ} \mathrm{C}$. Serial dilutions of ENL were made from stock solutions with cell culture medium. DMSO was used as negative control.

\section{Cell proliferation assay}

Cell proliferation was determined using the MTT assay. For the MTT assay, $5 \times 10^{3}$ cells/well were cultured in 96 -well plates and incubated in standard culture 
medium overnight. In $24 \mathrm{~h}$, the old medium was replaced with fresh medium containing ENL. After incubation for $24 \mathrm{~h}$, the MTT assay was performed as previously described (Chen et al. 2007). Data are presented from 3 separate experiments and the percentage of ENL-induced cell growth inhibition was determined using DMSO-treated cells (control) as the denominator.

\section{Cell cycle analysis}

Propidium iodide staining was performed by flow cytometry to analyze the cell cycle of WPMY-1 cells arrested by ENL. The cells at 40-50\% confluence were allowed to grow in the standard culture medium overnight and then transferred to serum-free medium. In $24 \mathrm{~h}$, the old medium was replaced with fresh medium containing ENL. After incubation for $24 \mathrm{~h}$, the cells were collected and prepared for flow cytometric analysis as described previously (Chen et al. 2007). The cell cycle distribution was analyzed using the ModFit LT for Mac V1.01 software.

\section{RNA extraction and real-time PCR}

Total RNA was isolated from cells using TRIzol (Invitrogen, La Jolla, CA, USA) according to the manufacturer's protocol. Reverse transcription was used the RT2 First Strand Kit and MMLV-RT (Takara, Dalian, China). The cDNA was subjected to real-time PCR amplification using gene specific primers and $2 \times$ Brilliant II SYBR Green QPCR Master Mix (Invitrogen) as described previously (Zhao et al. 2012). Specific primer pairs for GPER: forward primer

5'-TCTCTAACCTCCGCAACCAC-3' and reverse primer 5'-CTGGGGGTGGAGACAAGCAT-3'. Primer pairs for Actin: forward primer 
5'-ACTATCGGCAATGAGCGGTTCC-3' and reverse primer

5'-AGCACTGTGTTGGCA TAGAGGTC -3'. Real-time PCR was carried

out on an ABI 7500 thermocycler (Applied Biosystems, CA, USA). Specific gene

expression was calculated using the comparative $2^{-\Delta \Delta \mathrm{C} \text { T }}$ method with Actin as the calibrator.

\section{siRNA transfection}

WPMY-1 cells were plated in 6 -well plates $\left(2 \times 10^{5}\right.$ cells/well $)$ and transfected with 1nM siRNA with TurboFect Transfection Reagents (Thermo Fisher Scientific, Shanghai, China) following the manufacturer's instructions. Transfected Cells were cultured for $24 \mathrm{~h}$ and total mRNA was extracted using TRIzol for real-time PCR to check the siRNA knockdown effect or cells was planted in 96-well plate to examine the proliferation by MTT assay. Control siRNA (scrambled siRNA) and GPR30 siRNA were provided by Santa Cruz Biotechnology ( Cat. No. sc-37007, sc-60743).

\section{Immunoblotting}

Proteins extraction and immunoblotting were performed as described previously (Chen et al. 2007)

The protein concentration was determined using the Brandford assay (Sigma). About $60 \mu \mathrm{g}$ of protein extracts were separated by SDS-PAGE and transferred to a nitrocellulose membrane. The membranes were incubated with target antibodies. Protein bands were then detected by incubation with horseradish peroxidaseconjugated antibodies and visualized through an enhanced chemiluminescence reagent (Perkin-Elmer, Shanghai Divison, China). Actin or Tublin were used as 
loading control.

\section{Animals}

A total of 32 adult male Wistar rats ( 8 weeks old, 4 rats per standard cage), weighing about 200g were obtained from Laboratory Animal Center of Chinese Academy of Sciences (Shanghai, China). Animals were housed with food (standard rodent chow diet from Shuangshi animal feed Technology Co. Ltd, Suzhou, China) and water available ad libitum and kept in controlled room temperature $\left(22 \pm 2^{\circ} \mathrm{C}\right)$ and humidity (60-80\%) under a $12 \mathrm{~h} / 12 \mathrm{~h}$ light/dark cycle. Animal care procedures and experiments were conducted following the guidelines of the Care and Use of Experimental Animals (available from the Canadian Council on Animal Care, on their Web site at www.ccac.ca) and were approved by the Soochow University Animal Committee.

\section{Induction of BPH and treatments}

$\mathrm{BPH}$ induction and treatment were performed as follows. Briefly, 32 male Wistar rats were randomly divided into 4 groups (8 rats per group): (1) untreated control; (2) subcutaneous injection with TP (2mg/rat/d) to induce prostate enlargement; (3) TP-treated group intragastrical administrated with SDG (5mg/rat/d); and (4) TP-treated group intragastrical administrated with Finasteride (a positive control for experimental drugs in BPH studies, $0.1 \mathrm{mg} / \mathrm{rat} / \mathrm{d})$. The dose of TP was determined according to other report(Bisson et al. 2014; Wang et al. 2015). After acclimatization for 7 days, TP were administered once daily for 28 days to induce 
BPH and treatment phase continued for 28 days with TP alone, TP/SDG and TP/Finasteride (reference to Figure 1). The weights of rats were recorded weekly. On day 56, rats were weighed and sacrificed under anesthesia; whole prostates were immediately removed, weighed and snap frozen. Alternate prostrate lobes were immersed in 4\% paraformaldehyde for future histological analysis. Prostatic index (PI) by the following formula: PI = gross wet weight of prostate/weight of whole animal

\section{Histological and immunohistochemical studies}

Tissues were post-fixed in 4\% paraformaldehyde overnight and were cut in a cryostat at $14 \mu \mathrm{m}$. One section was stained with Haematoxylin and eosin (H\&E) for histological examination, and other sections were processed for immunofluorescence (IF) staining.

For H\&E staining, the sections were stained with Harris-modified hematoxylin solution for $8 \mathrm{~min}$, then rinsed with tap water for $10 \mathrm{~min}$. Next, the sections were put in $1 \%$ hydrochloric acid-alcohol solution for $30 \mathrm{sec}$ followed by washing with distilled water for $1 \mathrm{~min}$. Subsequently, sections were put in $0.2 \%$ ammonia water for 30-60 sec, and then the sections were washed again with distilled water for $5 \mathrm{~min}$. Finally, the sections were counterstained with eosin-phloxine solution for 30-60 sec and washed with 10 drops of $95 \%$ alcohol. Following dehydration with absolute alcohol and 95\% alcohol twice for 5 min each time, the samples were cleared twice in xylene (5 min each time) and mounted with xylene-based mounting medium. 
For IF staining, the sections were rinsed three times in PBS. Non-specific interactions were blocked by normal donkey serum in $0.3 \%$ Triton $\mathrm{X}-100$ for 1 hour, and then sections were incubated with primary GPR30 antibody at 1/500 ( Cat. No : sc-48525-R; Santa Cruz Biotechnology) at $4^{\circ} \mathrm{C}$ overnight. After washing, sections were incubated with fluorescein isothiocyanate (FITC)-labelled secondary antibody at 1/1000 (donkey anti-rabbit IgG-FITC; sc-2090; Santa Cruz Biotechnology) for 1 hour at room temperature. After rinsed with PBS for three times and sections were then stained with 4',6-diamidino-2-phenylindole (DAPI; Sigma, St. Louis, MO, USA) for 10 minutes. After mounting, the stained sections were observed using fluorescence microscope (Carl Zeiss, Jena, Germany).

\section{Statistical Analysis}

One-way ANOVA was used to test statistical differences among treatment groups followed by Tukey's multiple comparisons. All experiments in the present study were done at least thrice, and the results were expressed as the mean $\pm \mathrm{SE}$ and considered significant when $P<0.05$. 


\section{Results}

\section{ENL may bind to GPER}

Based on chemical structure similarity between G1 and ENL (Figure 2B,C), we performed docking simulation analyses to evaluate the plausible binding mode between ENL and GPER. Due to the limited X-ray solved crystal structures, Bovine rhodopsin (PDB code 1U19), which shares an identity of $40 \%$ amino acid sequence with GPER, was used for building the homology model of GPER. In order to investigate the binding sites of G1 and ENL to GPER, we used the CDOCKER module in Discovery Studio 4.0.

The docking results on 14 ns GPER conformer (Figure 2D, E) show that G1 and ENL pose a similar binding pose and both reached the amino acid residues Val 242, His 331, Trp 301, Thr 249, Cys 230, Ser 309, Val 306, Asn 305. The results showed that non-covalent bonds play a key role in G1 and ENL binding to GPER. From the $2 \mathrm{D}$ interaction map, it was found that the atom $\mathrm{O}$ at ENL form a tight hydrogen bond with GPER through the Asn 305, while atom Br at benzene of G1 interacts with GPER at Leu242 through a hydrophobic bond.

\section{ENL caused cell growth inhibition through a GPER-mediated mechanism}

In order to examine the effects of ENL on WPMY-1 cells proliferation, cells were treated for $24 \mathrm{~h}$ with different doses $(0-20 \mu \mathrm{M})$ of ENL. As shown in Figure 3A, ENL significantly inhibited the cell growth with concentrations $\geq 5 \mu \mathrm{M}$. Next, since the treatment of ENL produced a significant cell growth reduction, we investigated if ENL blocked cell cycle or induced cell apoptosis. Findings of flow cytometry 
indicated that treatment of the cells with ENL $(20 \mu \mathrm{M})$ for $24 \mathrm{~h}$ made the cells arrest in the G0/G1 phase $(59.7 \%$; Figure 3B; $\mathrm{P}<0.05)$, when comparing with control (50.8\%). Similarly, The GPER agonist G1 also blocked in cell cycle in the G0/G1 phase $(63.9 \%$; Figure 3B; $\mathrm{P}<0.05)$. The sub-G0/G1 (apoptotic cells) contents were not detectable even after an extended treatment time $(72 \mathrm{~h})$ and the cells were still blocked in the G0/G1 phase (data not shown).

Furthermore, we detected that both G1 and ENL treatment affected the expression of proteins involved in the cell cycle regulation. As shown in Figure 4, WPMY-1 cells treated with ENL or G1 showed a reduced cyclin D1 expression concomitantly with p53 and p21 upregulation. It has been demonstrated ERK is one of the important downstream target of GPER (Meyer et al. 2014). We found that treatment with ENL showed a marked increase of ERK1/2 phosphorylation after treatment for $24 \mathrm{~h}$ (Figure 4).

To further demonstrate the requirement of GPER in the inhibitory effect of ENL on cell proliferation, we knocked down GPER expression by siRNA against GPER and performed cell proliferation examination. We found that the cell proliferation rate increased from $58.2 \%$ (ENL group) to $82.3 \%$ (ENL plus GPER-siRNA). It suggested that the reduction in GPER expression was able to impair partially the growth inhibitory effects exerted by ENL(Figure 3C, D). 


\section{SDG inhibited the enlargement of BPH induced by testosterone propionate}

As shown in Figure 5A, there were no statistical differences of body weight of rats between the four treatment groups at the end of the study. Rat prostates were harvested from the blank control group, the TP-induced model group (Model), the SDG group and the positive control finasteride group (Fin). The mean total wet weight of the prostates in the model group, SDG group and finasteride group were $561 \mathrm{mg}, 424 \mathrm{mg}$ and $378 \mathrm{mg}$, respectively (Figure 5B). The total weigh of wet prostate and PI of the SDG group and finasteride group significantly decreased comparing with the model group (Figure 5B,C). These results indicated that SDG significantly inhibited the enlargement of the rat prostate and reduce PI in the rat BPH model.

H\&E staining showed that there were clear differences in the alveolar structure between TP-induced BPH model group and SDG treated group. The number of papillary projections of prostatic alveolus and thickness of the pseudostratified epithelial cells and stromal cells reduced in SDG treated group when comparing with the model group (Figure 5D-G).

We also checked the expression of GPER by real-time PCR and immunofluorescence staining in prostatic tissue; results consistently showed that SDG stimulated the levels of GPER. This result further implied that the inhibitory effect of SDG on BPH might be associated with the up-regulation of GPER by SDG (Figure 5H-K). 


\section{Discussion}

Several studies using preclinical BPH animal models (Bisson et al. 2014; de Amorim Ribeiro et al. 2016) and clinical trials (Simons et al. 2015; Zhang et al. 2008) showed that flaxseed and its derivative SDG exerted inhibitory effects on BPH. However, the underlying mechanisms are unclear. In the present study, we demonstrated that ENL, the mammalian metabolite of SDG, may bind to and activate GPER and lead to the sustained activation of ERK, one of the downstream target of GPER and finally hindered the proliferation of prostatic stromal WPMY-1 cells. ENL blocked WPMY-1 cells in the G0/G1 phase as a consequence of the up-regulation in cell cycle negative regulators such as p21 and p53 and a reduction in cyclin D1 expression, one the most important proteins involved in cell cycle progression.

The chemical structure of ENL shares similarity with estrodiol. It is logical to assume that ENL may bind to estrogen receptor. To date, estrogen receptors belonging to two distinct receptor families have been described: estrogen receptors(ER), ER $\alpha$ and ER $\beta$ and GPER, a member of the G-protein coupled receptor superfamily. ER $\alpha$ and ER $\beta$ are found predominantly in the nucleus and perform gene expression function (termed genomic effects). GPER are associated with rapid cellular signaling (termed non-genomic effects) (Hammes and Levin 2007). Previously, we demonstrated that ENL suppressed IGF-1 induced proliferation of prostate cancer cells by inhibiting its downstream AKT phosphorylation (Chen et al. 2009). This suggested that ENL could 
initiate rapid, non-genomic effects. Indeed, our docking simulation analyses showed that ENL positioned within the GPER-binding site and displayed a good affinity for GPER. Moreover, ENL reached the same binding site and shared similar molecular recognition properties to those observed for G1, the agonist of GPER. Stimulation of GPER induces the release of heparin-binding EGF, which binds and activates EGFR (Prenzel et al. 1999), leading to activation of downstream signaling molecules, such as ERK1/2 (Gaudet et al. 2015). It is well known that phosphorylated ERK1/2 translocate from the cytoplasm to the nucleus to phosphorylate their nuclear targets for transcriptional regulation (Chen et al. 1992). It has also been proposed that signal duration of ERK1/2 activation could decide a different cell-fate, with transient ERK1/2 activation closely related to cell survival and proliferation and conversely, sustained activation of ERK1/2 transmitting antiproliferative signals (Marshall 1995; Tang et al. 2002). In our study, we examined that ENL or G1, the agonist of GPER activated ERK1/2 lasting for $24 \mathrm{~h}$ and significantly inhibited the proliferation of WPMY-1 and induced G0/G1 cell-cycle arrest. Several previous studies also showed that GPER activation was associated with decreased cell proliferation through sustained activation of ERK1/2. For example, study by Chan et al. showed that activation of GPER inhibited the growth of prostate cancer cells through sustained activation of ERK1/2, upregulation of $\mathrm{p} 21$, and induction of $\mathrm{G}(2)$ cell-cycle arrest (Chan et al. 2010). Chimento et al. found that selective GPER activation by G1 decreased proliferation and activated apoptosis in tumor Leydig cells (Chimento et al. 2013). It is well established that accumulation of the p53 tumor suppressor protein 
have been shown to arrest cell cycle in G1 phase. p53-induced expression of p21 binds to the cyclins/CDKs, leads to inhibition of cyclins/CDKs activity, resulting in G1 cell cycle arrest (Tashiro et al. 2007). While decreasing cyclin D1 expression also inhibits the cyclins/CDKs activity and hinds cell cycle progression (Tashiro et al. 2007). In our study, the G0/G1 cell cycle arrest effects of ENL was further confirmed by increased expression of p53, p21 and decreased expression of cyclin D1 by ENL treatment.

Although we demonstrated that ENL may bind and activate the GPER/ERK signaling and further blocked the proliferation of human prostatic stromal cell line, WPMY-1. Our in vivo data again showed that SDG treatment activated the GPER activation and inhibited the enlargement of BPH. However, we can't exclude the possibility that other mechanisms that involved in the anti-BPH effects of ENL and SDG. ER $\alpha$ is highly expressed in the prostate in both epithelial and stromal cells (Royuela et al. 2001). A recent study has shown that an imbalance of the androgen level and enhanced estrogenic effects were the main cause of BPH (Farnsworth 1999). It had been demonstrated the ability of purified lignans to compete with estradiol, stimulate sex hormone binding globulin production, and inhibit steroid binding (Thompson 1998). Thus, regulation of classical hormonal functions by lignan may be the other mechanisms.

At last, we tested the effects of SDG on BPH growth by in vivo experiments. SDG significantly inhibited the growth of prostate induced by TP, recovered the tissue morphology. We need to point out that we did not test the dose dependent effect in our 
in vivo experiment and the dose of SDG $(5 \mathrm{mg} / \mathrm{rat} / \mathrm{d})$ is relative high when comparing with Zhang's human clinical trial (the doses are $300 \mathrm{mg}$ and $600 \mathrm{mg}$ per day) (Zhang et al. 2008). Further experiments with small doses of SDG are warranted.

Taken together, the results of the present study provide the new evidence that lignan suppressed BPH both in vitro and in vivo. ENL inhibited WPMY-1 cell growth by the induction of G0/G1 cell cycle arrest. ENL-induced cell cycle arrest may be mediated by the activation of GPER/ERK pathway and subsequent up-regulation of p53 and p21, down-regulation of cyclin D1. This work, in tandem with previous studies, will enhance our knowledge regarding the mechanism(s) of dietary phytochemicals on $\mathrm{BPH}$ prevention and ultimately expand the scope of adopting alternative approaches in $\mathrm{BPH}$ prevention.

Acknowledgments: This work was supported by grants from Natural Science Foundation of Jiangsu Province (BK20140372), Postgraduate Innovation Cultivating Project in Jiangsu Province (SJLX_0572).

Conflict of Interest: The authors declare no conflict of interest. 


\author{
Reference List
}

Azrad, M., Vollmer, R. T., Madden, J., Dewhirst, M., Polascik, T. J., Snyder, D. C., Ruffin, M. T., Moul, J. W., Brenner, D. E. and Demark-Wahnefried, W. 2013. Flaxseed-derived enterolactone is inversely associated with tumor cell proliferation in men with localized prostate cancer. J. Med. Food. 16(4):357-360.

Bisson, J. F., Hidalgo, S., Simons, R. and Verbruggen, M. 2014. Preventive effects of lignan extract from flax hulls on experimentally induced benign prostate hyperplasia. J. Med. Food. 17(6):650-656.

Borriello, S. P., Setchell, K. D., Axelson, M. and Lawson, A. M. 1985. Production and metabolism of lignans by the human faecal flora. J. Appl. Bacteriol. 58(1):37-43.

Chan, Q. K., Lam, H. M., Ng, C. F., Lee, A. Y., Chan, E. S., Ng, H. K., Ho, S. M. and Lau, K. M. 2010. Activation of GPR30 inhibits the growth of prostate cancer cells through sustained activation of Erk1/2, c-jun/c-fos-dependent upregulation of p21, and induction of $\mathrm{G}(2)$ cell-cycle arrest. Cell. Death. Differ. 17(9):1511-1523.

Chen, L. H., Fang, J., Li, H., Demark-Wahnefried, W. and Lin, X. 2007. Enterolactone induces apoptosis in human prostate carcinoma LNCaP cells via a mitochondrial-mediated, caspase-dependent pathway. Mol. Cancer. Ther. 6(9):2581-2590. 
Chen, L. H., Fang, J., Sun, Z., Li, H., Wu, Y., Demark-Wahnefried, W. and Lin, X. 2009. Enterolactone inhibits insulin-like growth factor-1 receptor signaling in human prostatic carcinoma PC-3 cells. J. Nutr. 139(4):653-659.

Chen, R. H., Sarnecki, C. and Blenis, J. 1992. Nuclear localization and regulation of erk- and rsk-encoded protein kinases. Mol. Cell. Biol. 12(3):915-927.

Chimento, A., Casaburi, I., Bartucci, M., Patrizii, M., Dattilo, R., Avena, P., Ando, S., Pezzi, V. and Sirianni, R. 2013. Selective GPER activation decreases proliferation and activates apoptosis in tumor Leydig cells. Cell. Death. Dis. 4:e747.

de Amorim Ribeiro, I. C., da Costa, C. A., da Silva, V. A., Correa, L. B., Boaventura, G. T. and Chagas, M. A. 2016. Flaxseed reduces epithelial proliferation but does not affect basal cells in induced benign prostatic hyperplasia in rats. In Press

Demark-Wahnefried, W., George, S. L., Switzer, B. R., Snyder, D. C., Madden, J. F., Polascik, T. J., Ruffin, M. T. t. and Vollmer, R. T. 2008a. Overcoming challenges in designing and implementing a phase II randomized controlled trial using a presurgical model to test a dietary intervention in prostate cancer. Clin. Trials. 5(3):262-72.

Demark-Wahnefried, W., Polascik, T. J., George, S. L., Switzer, B. R., Madden, J. F., Ruffin, M. T. t., Snyder, D. C., Owzar, K., Hars, V., Albala, D. M. and others. 2008b. Flaxseed supplementation (not dietary fat restriction) reduces prostate cancer proliferation rates in men presurgery. Cancer. Epidemiol. Biomarkers. 
Prev. 17(12):3577-87.

Demark-Wahnefried, W., Price, D. T., Polascik, T. J., Robertson, C. N., Anderson, E. E., Paulson, D. F., Walther, P. J., Gannon, M. and Vollmer, R. T. 2001. Pilot study of dietary fat restriction and flaxseed supplementation in men with prostate cancer before surgery: exploring the effects on hormonal levels, prostate-specific antigen, and histopathologic features. Urology. 58(1):47-52.

Demark-Wahnefried, W., Robertson, C. N., Walther, P. J., Polascik, T. J., Paulson, D. F. and Vollmer, R. T. 2004. Pilot study to explore effects of low-fat, flaxseed-supplemented diet on proliferation of benign prostatic epithelium and prostate-specific antigen. Urology. 63(5):900-4.

Farnsworth, W. E. 1999. Estrogen in the etiopathogenesis of BPH. Prostate. 41(4):263-74.

Filardo, E. J., Quinn, J. A., Bland, K. I. and Frackelton, A. R., Jr. 2000. Estrogen-induced activation of Erk-1 and Erk-2 requires the G protein-coupled receptor homolog, GPR30, and occurs via trans-activation of the epidermal growth factor receptor through release of HB-EGF. Mol. Endocrinol. 14(10):1649-1660.

Gaudet, H. M., Cheng, S. B., Christensen, E. M. and Filardo, E. J. 2015. The G-protein coupled estrogen receptor, GPER: The inside and inside-out story. Mol. Cell. Endocrinol. 418 Pt 3:207-219.

Gencel, V. B., Benjamin, M. M., Bahou, S. N. and Khalil, R. A. 2012. Vascular effects of phytoestrogens and alternative menopausal hormone therapy in 
cardiovascular disease. Mini. Rev. Med. Chem. 12(2):149-174.

Hammes, S. R. and Levin, E. R. 2007. Extranuclear steroid receptors: nature and actions. Endocr. Rev. 28(7):726-741.

Isaacs, J. T. 1994. Etiology of benign prostatic hyperplasia. Eur. Urol. 25 Suppl $1: 6-9$

Lappano, R., Rosano, C., Santolla, M. F., Pupo, M., De Francesco, E. M., De Marco, P., Ponassi, M., Spallarossa, A., Ranise, A. and Maggiolini, M. 2012. Two novel GPER agonists induce gene expression changes and growth effects in cancer cells. Curr. Cancer. Drug. Targets. 12(5):531-542.

Marshall, C. J. 1995. Specificity of receptor tyrosine kinase signaling: transient versus sustained extracellular signal-regulated kinase activation. Cell. 80(2): 179-185.

Meyer, M. R., Fredette, N. C., Howard, T. A., Hu, C., Ramesh, C., Daniel, C., Amann, K., Arterburn, J. B., Barton, M. and Prossnitz, E. R. 2014. G protein-coupled estrogen receptor protects from atherosclerosis. Sci. Rep. 4:7564.

Pan, A., Yu, D., Demark-Wahnefried, W., Franco, O. H. and Lin, X. 2009. Meta-analysis of the effects of flaxseed interventions on blood lipids. Am. J. Clin. Nutr. 90(2):288-297.

Prenzel, N., Zwick, E., Daub, H., Leserer, M., Abraham, R., Wallasch, C. and Ullrich, A. 1999. EGF receptor transactivation by G-protein-coupled receptors requires metalloproteinase cleavage of proHB-EGF. Nature. 
402(6764):884-888.

Ren, G. Y., Chen, C. Y., Chen, G. C., Chen, W. G., Pan, A., Pan, C. W., Zhang, Y. H., Qin, L. Q. and Chen, L. H. 2016. Effect of Flaxseed Intervention on Inflammatory Marker C-Reactive Protein: A Systematic Review and Meta-Analysis of Randomized Controlled Trials. Nutrients. 8(3):136.

Royuela, M., de Miguel, M. P., Bethencourt, F. R., Sanchez-Chapado, M., Fraile, B., Arenas, M. I. and Paniagua, R. 2001. Estrogen receptors alpha and beta in the normal, hyperplastic and carcinomatous human prostate. J. Endocrinol. 168(3):447-454.

Simons, R., Sonawane, N., Verbruggen, M. and Chaudhary, J. 2015. Efficacy and safety of a flaxseed hull extract in the symptomatic management of benign prostatic hyperplasia: a parallel, randomized, double-blind, placebo-controlled, pilot study. J. Med. Food. 18(2):233-240.

Smith, L. C., Ralston-Hooper, K. J., Ferguson, P. L. and Sabo-Attwood, T. 2016. THE G Protein-Coupled Estrogen Receptor Agonist G-1 Inhibits Nuclear Estrogen Receptor Activity and Stimulates Novel Phosphoproteomic Signatures. Toxicol. Sci. 151(2):434-446

Tang, D., Wu, D., Hirao, A., Lahti, J. M., Liu, L., Mazza, B., Kidd, V. J., Mak, T. W. and Ingram, A. J. 2002. ERK activation mediates cell cycle arrest and apoptosis after DNA damage independently of p53. J. Biol. Chem. 277(15):12710-7.

Tashiro, E., Tsuchiya, A. and Imoto, M. 2007. Functions of cyclin D1 as an oncogene 
and regulation of cyclin D1 expression. Cancer. Sci. 98(5):629-635.

Thompson, L. U. 1998. Experimental studies on lignans and cancer. Baillieres. Clin. Endocrinol. Metab. 12(4):691-705.

Ventura, S., Oliver, V., White, C. W., Xie, J. H., Haynes, J. M. and Exintaris, B. 2011. Novel drug targets for the pharmacotherapy of benign prostatic hyperplasia (BPH). Br. J. Pharmacol. 163(5):891-907.

Wang, C., Du, X., Yang, R., Liu, J., Xu, D., Shi, J., Chen, L., Shao, R., Fan, G., Gao, X. and others. 2015. The prevention and treatment effects of tanshinone IIA on oestrogen/androgen-induced benign prostatic hyperplasia in rats. J. Steroid. Biochem. Mol. Biol. 145:28-37.

Wu, G., Robertson, D. H., Brooks, C. L., 3rd and Vieth, M. 2003. Detailed analysis of grid-based molecular docking: A case study of CDOCKER-A CHARMm-based MD docking algorithm. J. Comput. Chem. 24(13):1549-1562.

Wu, H., Pan, A., Yu, Z., Qi, Q., Lu, L., Zhang, G., Yu, D., Zong, G., Zhou, Y., Chen, X. and others. 2010. Lifestyle counseling and supplementation with flaxseed or walnuts influence the management of metabolic syndrome. J. Nutr. 140(11):1937-42.

Yu, Y., Song, J., Song, Y., Guo, X., Han, Y. and Wei, J. 2013. Characterization of catalytic activity and structure of selenocysteine-containing hGSTZ1c-1c based on site-directed mutagenesis and computational analysis. IUBMB. Life. 65(2):163-70. 
Zhang, W., Wang, X., Liu, Y., Tian, H., Flickinger, B., Empie, M. W. and Sun, S. Z. 2008. Effects of dietary flaxseed lignan extract on symptoms of benign prostatic hyperplasia. J. Med. Food. 11(2):207-214.

Zhao, L., Sun, Y., Hou, Y., Peng, Q., Wang, L., Luo, H., Tang, X., Zeng, Z. and Liu, M. 2012. MiRNA expression analysis of cancer-associated fibroblasts and normal fibroblasts in breast cancer. Int. J. Biochem. Cell. Biol. 44(11):2051-2059. 


\section{Figure legends}

Figure 1. Schematic figure for induction of BPH and treatments.

Figure 2. GPER docked with G1 and ENL. (A-C). chemical structure of SDG, ENL and G1. (D) residues involved in the interactions with G1. (E) residues involved in the interactions with ENL.

Figure 3. ENL inhibited the proliferation of WPMY-1 cells and blocks the cell cycle in the G0/G1 phase; knockdown of GPER impaired partially the growth inhibitory effects exerted by ENL. (A) the cells were incubated on a 96-well plate overnight. Then indicated doses of ENL were supplemented for $24 \mathrm{~h}$ and cell proliferation was checked by MTT assay. (B) the cells were cultured the same as (A); cell cycle were checked by flow cytometry assay. ENL $(20 \mu \mathrm{M})$, G1 $(1 \mu \mathrm{M})$. (C) after transfection, the cells were kept for incubation for $24 \mathrm{~h}$ followed by real-time PCR. (D) the transfected cells were incubated $24 \mathrm{~h}$ and distributed on 96-well plates. ENL $(20 \mu \mathrm{M})$ was supplemented and the incubation continued for $24 \mathrm{~h}$. Then, cell proliferation was checked by MTT assay. The untreated controls were set to $100 \%$. Values are means $\pm \mathrm{SE}, \mathrm{n}=6$. Means without a common letter differ, $P<0.05$.

Figure 4. ENL increased the expression of GPER, its downstream target ERK, and changed the expression of cell cycle related proteins. WPMY-1 cells were treated with ENL $(20 \mu \mathrm{M})$ and $\mathrm{G} 1(1 \mu \mathrm{M})$ for $24 \mathrm{~h}$. Total proteins were extracted and target 
proteins were examined by western-blot. (B) Quantified data of immunoblotting. The densitometry data of GPER, p-ERK, P53, P21 and Cyclin D1 obtained in (A) were normalized to Tublin or Actin levels and those of the control (lane 1). Values are means $\pm \mathrm{SE}, \mathrm{n}=4$. Means without a common letter differ, $P<0.05$.

Figure 5. ENL effectively inhibited TP-induced BPH in rats. (A) the total body weight of the rat prostates in each group after 28 days of treatment. (B) the wet weight of the rat prostates in each group. $n=8$. (C) quantitative analysis of the rat prostatic index of each group. $n=8$. (D-G) representative photomicrographs for H\&E staining of rat prostate tissues (magnification: 200), Scale bars: $50 \mu \mathrm{m}$. D for control group, E for model group, F for Fin treated group and G for SDG treated group. $(\mathrm{H})$ real-time PCR for GPER. $\mathrm{n}=4$. (I-K) representative photomicrographs for immunofluorescence staining for GPER of rat prostate tissues (magnification: 400), Scale bars: $50 \mu \mathrm{m}$. I for untreated control group (Normal), J for model group, $\mathrm{K}$ for SDG treated group.

Values are means \pm SE. Means without a common letter differ, $P<0.05$. 


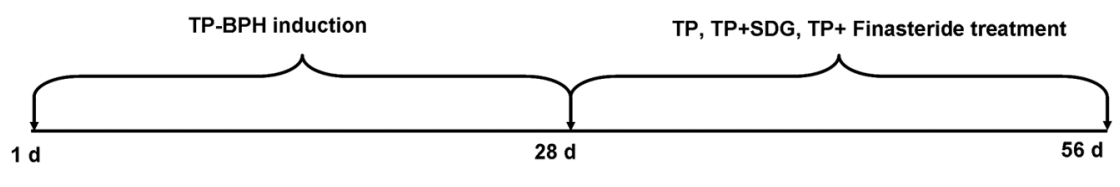

Figure 1. Schematic figure for induction of BPH and treatments.

$$
150 \times 20 \mathrm{~mm}(300 \times 300 \mathrm{DPI})
$$


A

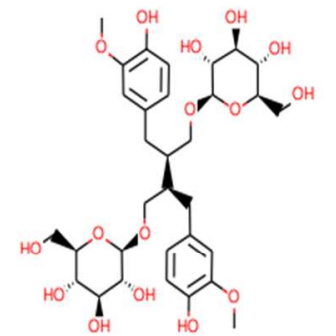

Secoisolariciresinol Diglucoside (SDG)

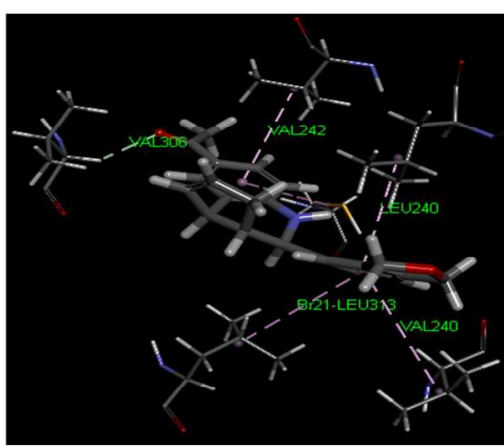

E

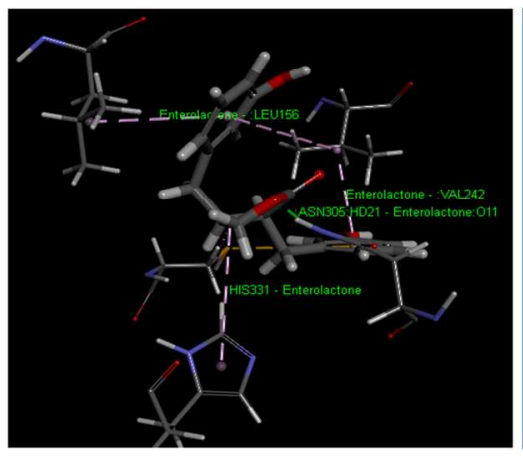

B

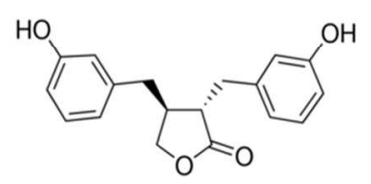

Enterolactone (ENL)

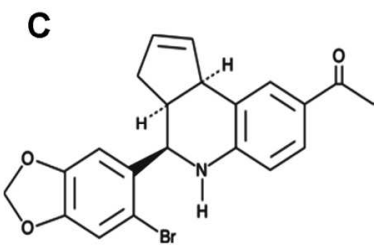

G1
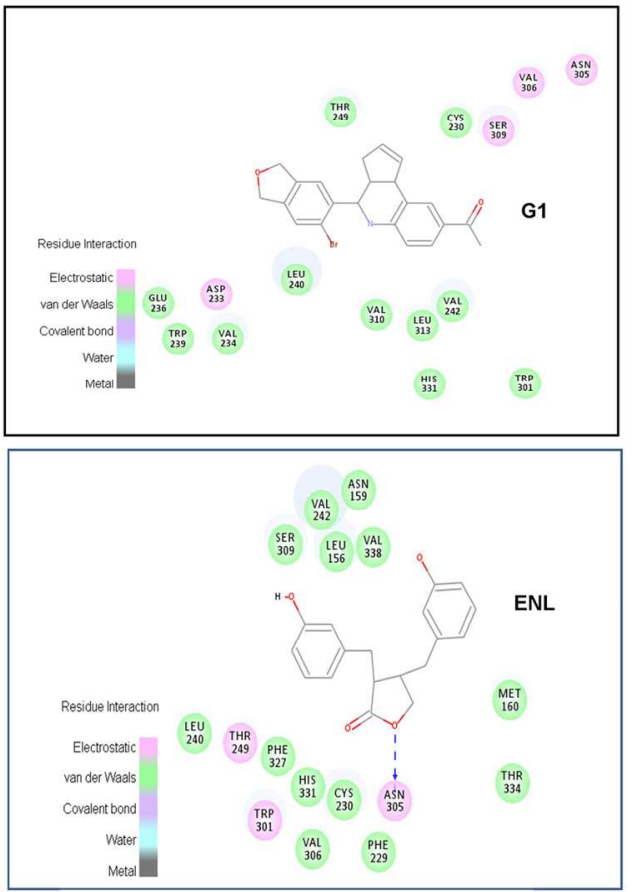

Figure 2. GPER docked with G1 and ENL. $170 \times 169 \mathrm{~mm}(300 \times 300 \mathrm{DPI})$ 
A

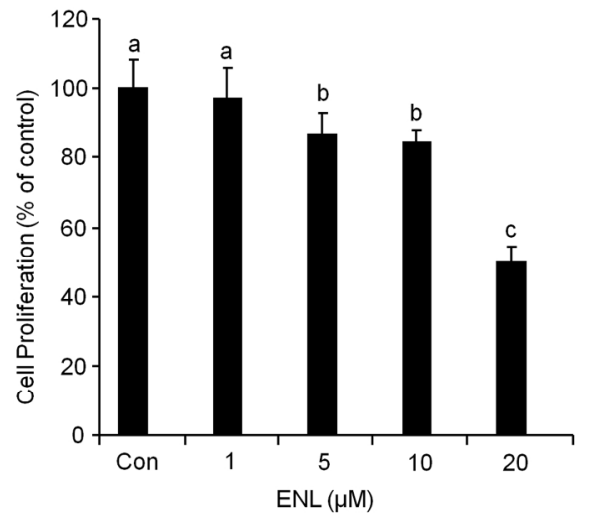

C

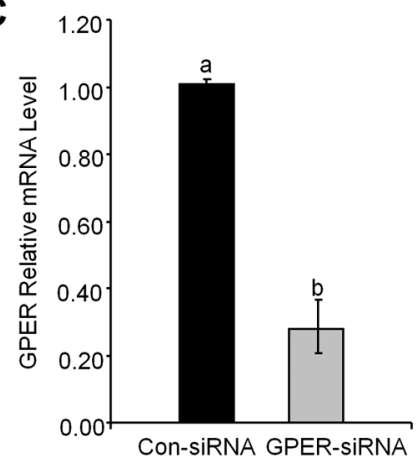

B
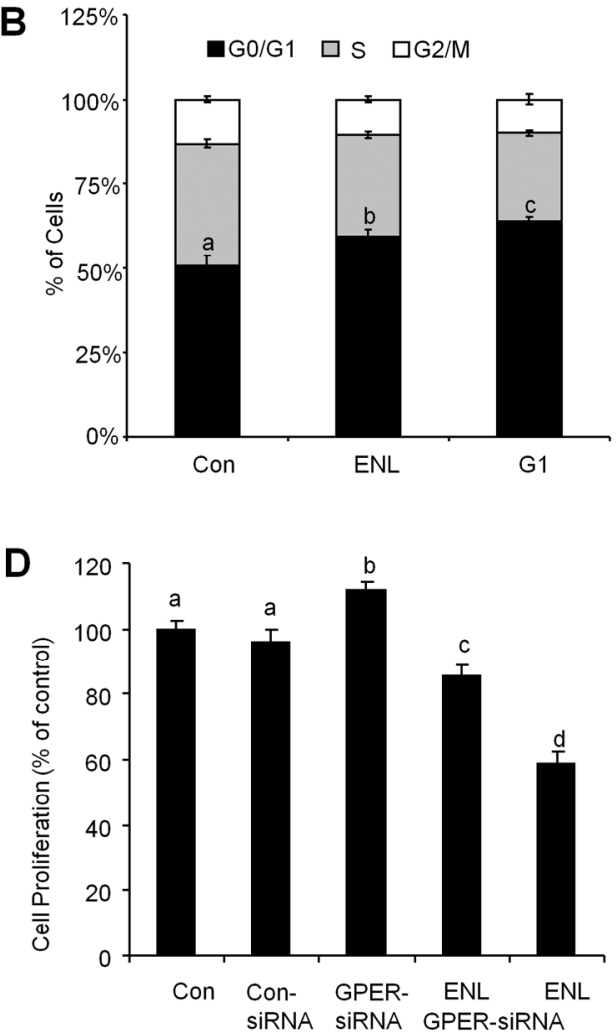

Figure 3. ENL inhibited the proliferation of WPMY-1 cells and blocks the cell cycle in the G0/G1 phase; knockdown of GPER impaired partially the growth inhibitory effects exerted by ENL. 

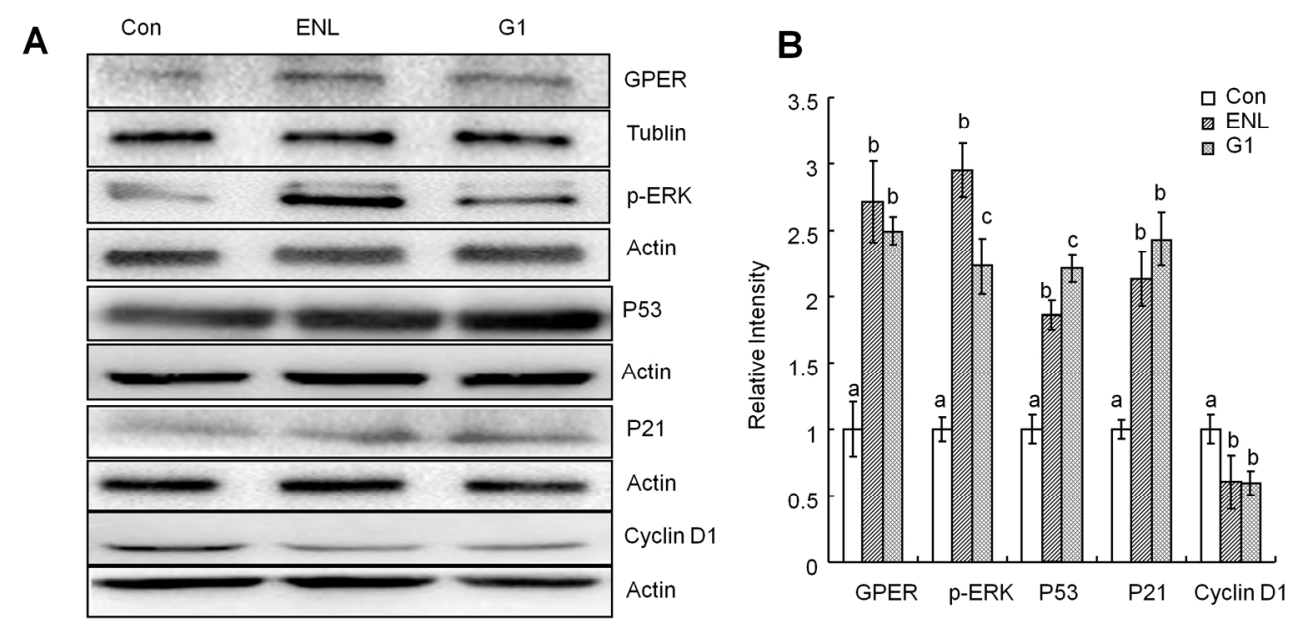

Figure 4. ENL increased the expression of GPER, its downstream target ERK, and changed the expression of cell cycle related proteins.

$172 \times 81 \mathrm{~mm}(300 \times 300$ DPI $)$ 
A

B C
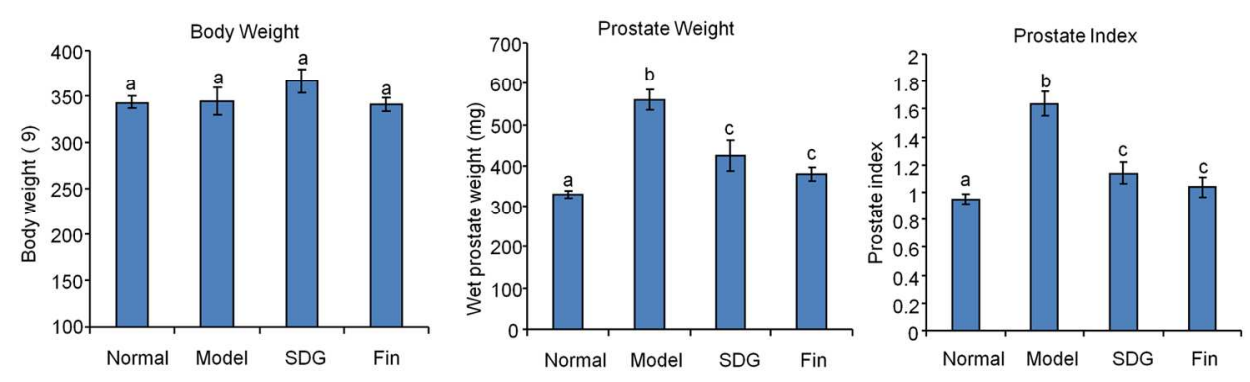

D

E

$\mathbf{F}$

G
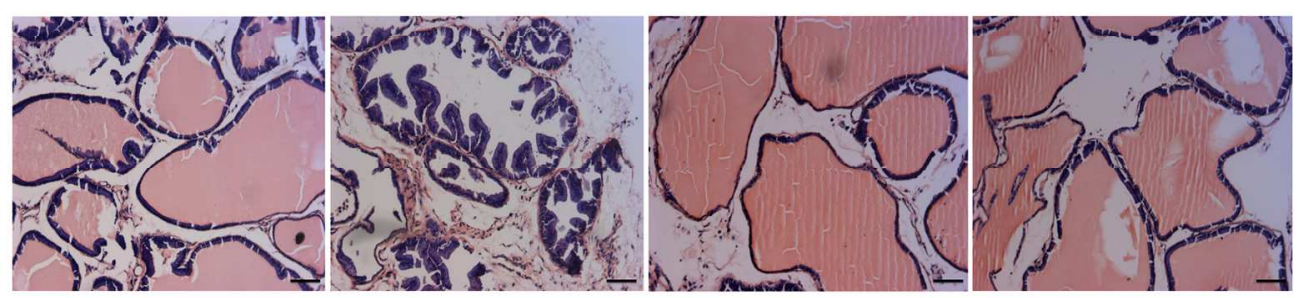

H

I

$\mathbf{J}$

K
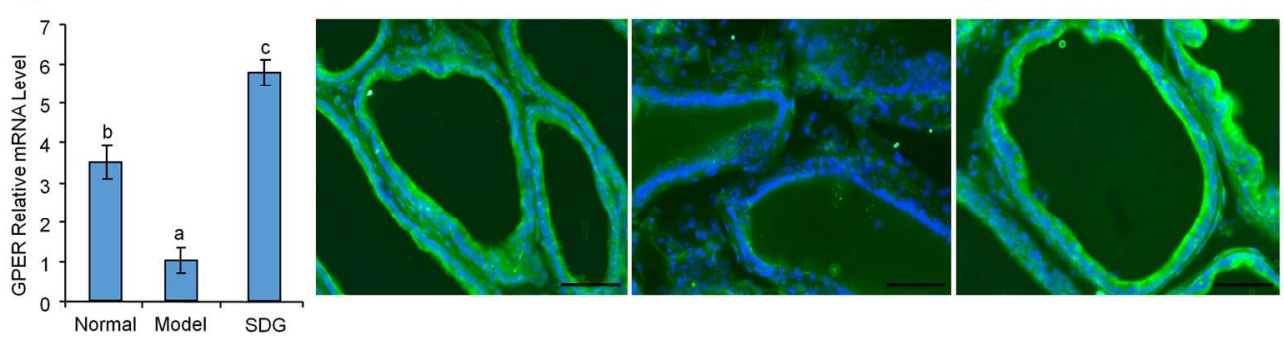

Figure 5. ENL effectively inhibited TP-induced BPH in rats.

$175 \times 159 \mathrm{~mm}(300 \times 300 \mathrm{DPI})$ 